\title{
LA DETERMINABILIDAD, DE FICHTE A DELEUZE, O LA THATHANDLUNG COMO AFIRMACIÓN DE LA DIFERENCIA
}

\author{
DETERMINABILITY, FROM FICHTE TO \\ DELEUZE, OR THE THATHANDLUNG AS \\ AFFIRMATION OF DIFFERENCE
}

\author{
JULIÁN FERREYRA* \\ Universidad de Buenos Aires / CONICET
}

Resumen: Este artículo se propone poner en relación el pensamiento de Johann Fichte con el de Gilles Deleuze, a partir del concepto de determinabilidad. El filósofo francés encuentra en Fichte uno de los antecedentes de su propio esfuerzo de alcanzar el punto de vista de la génesis de la experiencia a partir de la triplicidad de valores de la Idea: la determinación, lo indeterminado y lo determinable. Sin embargo, según Deleuze este enfoque se encuentra desnaturalizado en Fichte por un moralismo donde los tres valores aparecen fusionados. Intentaremos mostrar que, en lugar de traicionar el verdadero movimiento del pensamiento, el «moralismo» fichteano permite su concreción, justamente en los mismos términos en que Deleuze lo concibe. La inmanencia de los tres valores lógicos, y el especial énfasis otorgado al valor de lo determinable, hacen posible encontrar sólidas afinidades entre una filosofía de la diferencia -y no de la identidad-, y una fundada en una Thathandlung -y no en un sustrato-.

Palabras Clave: Deleuze, Fichte, determinabilidad, punto de vista de la génesis, Thathandlung.

*djulianferreyra@gmail.com. Investigador adjunto del CONICET y jefe de trabajos prácticos de la materia Antropología Filosófica (FFyL, Universidad de Buenos Aires). Director de los proyectos de investigación financiados "Del idealismo alemán a la filosofía francesa de la diferencia: tras las fuentes del poskantismo deleuziano" (UBCyT) y "Deleuze: ontología práctica" (Foncyt). 
АвsтRACT: This paper aims to establish a bridge between the thought of Johann Fichte and that of Gilles Deleuze, through the notion of determinability. The French philosopher regards highly the fact that Fichte reached the point of view of genesis of experience by conceiving three of values in the Idea: the determination, the indeterminate and the determinability. However, according to Deleuze this approach is denatured in Fichte by the fusion of all three values. We will try to show that, instead of betraying the truth movement of thought, the Fichtean «moralism» allows its achievement, precisely in the Deleuzian terms. The immanence of the three logical values, and the particular emphasis given to the determinable, making it possible to find sound affinities between a philosophy of difference -and not of identity-, and one grounded in the Thathandlug -and not in a substratum.

KeYwords: Deleuze, Fichte, determinability, point of view of genesis, Thathandlung.

\section{Introducción}

La proposición fundamental de la ontología de Gilles Deleuze es que la trama de todo lo que hay está constituida por la relación intrínseca de Ideas que, necesariamente, implican una triplicidad de valores lógicos: lo indeterminado, la determinación y lo determinable ${ }^{1}$. Estas páginas se proponen mostrar que esta proposición ontológica es válida del mismo modo, con el mismo nivel de importancia y centralidad, para Fichte. Sólo de esta manera Fichte logra escapar a las acusaciones de ser el acabamiento de la filosofía del sujeto de raigambre cartesiana, de acuerdo a las cuales su Yo como sujeto unitario de toda la realidad vendría a ocupar, como su equivalente, el lugar de Dios como sustancia absoluta. O, lo que es lo mismo, evitar que uno de los principales adversarios del dogmatismo ocupe en la historia de la filosofía el lugar del dogmático por excelencia. Deleuze, por su parte, logra escapar a las acusaciones de equivalencia de su fondo ontológico, esto es, a la paradoja de pensar a la filosofía de la diferencia como la filosofía de la indiferencia por excelencia.

De acuerdo a Deleuze, la verdadera revolución copernicana de Kant fue pensar que la Idea debe tener necesariamente tres valores: la determinación, lo indeterminado y lo determinable. Pero cometió el error de pensar los tres valores

1 El carácter fundamental de esta triplicidad en la ontología de Deleuze se observa a lo largo del capítulo 4 de Diferencia y repetición (Deleuze, 1968), dedicado a la teoría deleuziana de la Idea. Para una argumentación detallada, cfr. Ferreyra, 2012 y 2014 c. 
en una relación extrínseca ${ }^{2}$. De allí el escepticismo kantiano: Deleuze interpreta que en Kant el Yo determina el objeto a partir de sus formas a priori, pero nunca puede conocer la cosa en sí. El Yo posee la forma de la determinación, pero el origen de la materia indeterminada le resulta un misterio irresoluble. En esta trampa escéptica («empirista», dice Deleuze), el autor de Diferencia y repetición enfatiza la exterioridad de los términos. Por tanto, lo mismo da si tomamos la determinación como punto de partida a priori o si, en cambio, partimos de lo indeterminado. En este último caso lo misterioso será el origen de la determinación (por ejemplo, un Yo soy "indeterminado" que se iría determinando por causas extrínsecas). Incluso en el caso en que la certeza se aplique con la misma fuerza a lo indeterminado y la determinación, mientras permanezcan en relación extrínseca (por ejemplo, surgiendo de facultades diferentes), el problema se mantiene $^{3}$. Cuando la determinación y lo indeterminado se piensan como externos uno al otro, es necesario pensarlos como inmediatamente aplicables el uno a otro (el sinsentido cartesiano ${ }^{4}$ ), o postular un tercer término, deus ex machina, que nos permita ponerlos en relación: por ejemplo, el esquematismo en Kant, o la armonía preestablecida ${ }^{5}$. La trampa está en el problema y no en la solución: una vez que partimos desde términos concebidos como autónomos, ya no hay manera de conciliarlos más que el milagro.

Lo que Kant había mantenido exterior, dice Deleuze, los poskantianos lo sintetizan y unifican (Deleuze, 1968, 221). Rozan así el verdadero movimiento del pensamiento, según el cual «en la Idea [hay] una unidad objetiva problemática interna de lo indeterminado, de lo determinable y de la determinación» (Deleuze, 1968, 220). Deleuze caracteriza la filosofía de Fichte, en tanto poskantiano, como una unidad de lo indeterminado, de lo determinable y de la determinación. Nada más natural: no hay en Fichte nada exterior, todo es en y por el Yo. No sabemos si Deleuze accedió a esta interpretación de la filosofía de Fichte por intuición

2 En Kant, dice Deleuze, «dos de los tres momentos son caracteres extrínsecos (si la Idea es en sí misma indeterminada, no es determinable más que con respecto a los objetos de la experiencia, y no lleva el ideal de determinación más que respecto a los conceptos del entendimiento)» (Deleuze, 1968, 220-221).

3 Yo diría que los tres modelos pueden aplicarse a Kant, pero no es el punto aquí: son términos extrínsecos y eso es lo que está puesto en cuestión.

4 «El cogito es un sinsentido, en la medida en que esta proposición pretende decirse, tanto a sí misma como a su sentido» (Deleuze, 1968, 353).

5 Salvo que llamemos, como podría intentar hacerse, «armonía preestablecida» a la Thathandlung misma, al proceso intrínseco que entrelaza determinación, determinable e indeterminado. Así debería interpretarse la lectura que Deleuze hace de Leibniz y el paralelismo de Spinoza. 
intelectual, que se le reveló a los pistoletazos, como un pantallazo inmediato, o si surge de un análisis detallado de la obra. No lo sabemos porque Deleuze no desarrolla en absoluto esta afirmación en el caso particular de Fichte y se refiere a él en forma directa en pocas oportunidades ${ }^{6}$. No hace lo recomendable metodológicamente: luego de afirmar «los poskantianos conciben la Idea como una unidad objetiva problemática interna, de lo indeterminado, de lo determinable y de la determinación", pasa a argumentar: En el caso de Fichte, esto se observa de la siguiente manera, en Schelling de tal otra, en Hegel, en Maimon, en Novalis, etc. ${ }^{7}$ Deleuze sólo se explaya en forma por cierto oscura en el caso de Salomon Maimon, a quien considera el «fundador del poskantismo». Sin embargo, su interpretación, peculiar, oscura y potente permite fructíferos acercamientos a la obra de Fichte, Schelling y Hegel. En estas páginas, intentaré reponer el desarrollo implícito de la filosofía de Fichte que el poskantismo deleuziano supone, y mostrar los beneficios que otorga para la interpretación del pensamiento de ambos filósofos ${ }^{8}$.

La hipótesis interpretativa del Fichte de $1794^{9}$ que desarrollaré en lo que sigue es que el Yo como actuar, como «actuar sobre sí mismo» (Fichte, GA I/2, 313) es indeterminado, determinable y determinación ${ }^{10}$. Deleuze denomina a estos tres aspectos de la Idea «valores lógicos». Esta denominación hace tentador intentar correlacionarlos con los tres principios del Fundamento para toda la doctrina de la ciencia de 1794. Por ejemplo: el Yo absoluto podría ser la determinación, el no-Yo lo indeterminado y el límite lo determinable. Bello juego, pero que no es sin embargo la perspectiva más fructífera. No lo es, por una parte, porque otorga a los principios del Fundamento una jerarquía para la Doctrina de la ciencia en general de la cual carecen (de lo cual las distintas exposiciones

6 En Diferencia y repetición lo menciona directamente (y no como parte del "poskantismo») una sola vez, y la referencia remite a L'évolution et la structure de la Doctrine de la Science chez Fichte de Guéroult (para un estudio detallado de la presencia de Fichte en la obra de Deleuze, $c f$. Ferreyra, 2014a).

7 Recurro aquí a la lista exhaustiva de poskantianos que se puede reconstruir a lo largo de la obra y las clases de Deleuze.

8 La tarea de confrontar el pensamiento de Fichte con el de Deleuze tiene pocos antecedentes. Se destacan los esfuerzos de Goddard (1999, 2008), sobre los que volveremos más adelante. También están las referencias de Rametta (2010) en torno a la biopolítica y Acosta (2009) sobre la forma diferente en que cada uno piensa lo absoluto.

9 Tomaremos para este análisis textos publicados por Fichte entre 1794 y 1798, incluidos en los volúmenes I/2, I/3 y I/4 de la edición de la obra completa de Fichte realizada bajo la dirección de Lauth.

10 Sobre la relación entre la determinación, lo determinable y lo indeterminado en Fichte, cfr. Zöller, 1998, 89-93. 
de la misma que realiza Fichte a lo largo de su vida, prescindiendo de estos tres principios, es prueba suficiente). Por otra parte, no permite problematizar los aspectos centrales de la filosofía de Fichte, la naturaleza misma de la Doctrina de la ciencia.

Intentaremos pensar, mejor, a lo indeterminado, lo determinable y la determinación como aspectos de la Thathandlug, esa autoactividad o autogénesis que está en el corazón de la Doctrina de la ciencia misma, ese espíritu que no puede ser expresado en sí mismo sino mediante una exposición tan necesaria como necesariamente inadecuada (y por tanto infinita). Los principios son una expresión (Ausdruck) de esta «autogénesis que ni se da ni se puede dar entre las determinaciones empíricas de nuestra conciencia, sino que más bien es el fundamento de toda conciencia» (Fichte, GA I/2, 255 [1975, 13]). La cuestión no radica en los principios (ni siquiera en el Yo absoluto como "principio fundamental absolutamente primero») sino en la Thathandlug que expresan. Y no es, claramente, lo mismo expresar y ser. La correlación que pueda establecerse entre los tres principios y los valores deleuzianos es entonces posible como expresión de la autogénesis. Pero en estas páginas exploramos la posibilidad de que éstos sean la lógica propia de la autogénesis, y abran así la posibilidad de indagar el funcionamiento del fundamento de toda realidad, incluida nuestra conciencia. A partir de la intrinsecación de estos tres valores, podremos asomarnos a la forma en la cual Fichte no cesó en el intento de acceder a la Thathandlung como un fundamento último de la realidad que se identifica con esa realidad que funda, y resolver así «el desconcierto más sorprendente y ancestral» (Fichte, GA I/3, 176): cómo es posible que el yo sea absolutamente libre y limitado al mismo tiempo, finito e infinito, esto es necesariamente finito e infinito.

\section{El punto de vista de la génesis}

Como señalamos más arriba, el pasaje de Kant a los poskantianos está marcado de acuerdo a Deleuze por la unificación de los momentos de la Idea que Kant mantenía separados y extrínsecos. La importancia ontológica de este pasaje es considerable, ya que según Deleuze en él se deben «encontrar (quizás) las verdaderas razones por las cuales Kant, como los poskantianos le reprochan, se quedó en el punto de vista del condicionamiento sin alcanzar el de la génesis» (Deleuze, 1968, 221). En otras palabras, según Deleuze, Fichte alcanza el punto de vista de 
la génesis, y no se queda meramente en el condicionamiento ${ }^{11}$, en tanto piensa los tres valores en una unidad. El carácter genético de la filosofía de Fichte deriva del hecho de que su fundamento es simultáneamente indeterminado, determinable y determinación. Lo cual, siguiendo la hipótesis que guía estas páginas, quiere decir que el punto de vista de la génesis que, según Deleuze, alcanza, no es otra cosa que la Thathandlung, o el Yo como autoactividad ${ }^{12}$, Yo absoluto que no se confunde con el Yo = Yo del primer principio del Grundlage sino que es aquello que los tres principios expresan, y que está en su fundamento ${ }^{13}$.

El Yo produce (produciert) ${ }^{14}$, engendra (entsteht), porque en él la indeterminación, la determinabilidad y la determinación se encuentran en unidad objetiva problemática interna. Sólo así, sólo en tanto está constituido por la dinámica de los tres, es capaz de expresar adecuadamente la Thathandlung. Es cierto que Fichte dice que producir y determinar van siempre de la mano (Fichte, 1966, 187), con lo cual pareciera que la producción es un privilegio exclusivo del valor «determinación». Pero «determinar» es un proceso que no debe confundirse, justamente, con una mera determinación. Porque un mero poder de determinación, por una parte, "pone el límite como sólido, fijo e inmutable» (Fichte, GA $\mathrm{I} / 2,359-360)^{15}$. Y, por otra parte, depende de que lo indeterminado sea dado. La determinación es así condición de la experiencia posible, pero nunca principio genético. Hay un límite fijo entre la determinación y lo indeterminado que ésta debe determinar. Los términos permanecen extrínsecos, y lo determinable aparece, como señalamos más arriba, como mero medio para encarnar la armonía preestablecida. Superar esa perspectiva es el punto que Deleuze reconoce a Fichte. Lo determinable no es el mediador entre estos dos planos escindidos. El oscilar (schweben) no supone los términos entre los cuales oscila sino que los produce. A las objeciones escépticas de Maimon, Fichte responde: «la imaginación [no es

11 Este pasaje es casi textualmente reconocido por Fichte: «Según Kant, toda conciencia está condicionada sólo por la autoconciencia (...) Según la Doctrina de la ciencia, toda conciencia está determinada por la autoconciencia», Fichte, 1970, 229.

12 Breazeale utiliza la oposición reflexiva entre determinación y determinable para explicar la actividad característica del Yo $(2013,55-56)$.

13 Dado que nos limitamos al período 1794-1798, es el Yo y no Dios el concepto central.

14 «Originariamente el Yo produce el objeto» (Fichte, 1966, 180).

«Si entre los opuestos (...) se pone el límite como sólido, fijo, e inmutable, entonces los dos términos son conciliados por determinación, pero no por determinabilidad (...) La determinabilidad es la Idea -inalcanzable por este camino- de la determinación» (Fichte, 1964, 359-360). 
un intermediario entre las leyes del pensamiento y los objetos] sino que produce el objeto mismo» (Fichte, GA I/3, 190).

La génesis exige la abolición de un límite fijo entre lo determinado y lo indeterminado. Pero eso no hace verdadera la proposición opuesta: engendrar no significa tampoco un mero fluir, un mero oscilar perpetuo. Tomemos la analogía con la de un producto de la naturaleza que utiliza Fichte en su Fundamento del derecho natural. Se trata de dar lugar a un forma capaz de perdurar y no a una eterna lucha entre el ser y el no-ser (Fichte, GA I/4, 19). La permanencia implica una interrupción en el constante operar de la fuerza formadora de la naturaleza.

[Si la acción fuera continua] se encontraría en la naturaleza un tránsito constante hacia otras formas naturales, pero nunca una permanencia de la misma forma natural; un devenir eterno, pero nunca un ser. Y puesto que no existiría nada que pudiera transformarse, tampoco sería posible el transformarse como tal (Fichte, GA I/4, 96).

En un continuo oscilar no habría génesis, sino un puro triunfo de lo indeterminado ${ }^{16}$. Los otros valores lógicos permiten que las cosas sean. La Idea no reúne sin distinguir, sin determinar lo indeterminado bajo la forma de lo determinable. De lo contrario habría devenir, pero nunca ser. Y puesto que no habría nada que pudiera engendrarse, tampoco existiría la génesis como tal.

\section{Aniquilación de la génesis en lo absoluto}

Sin embargo, Deleuze considera que Fichte no roza el verdadero movimiento del pensamiento sin traicionarlo. Es decir, no alcanza el punto de vista de la génesis sin dejar, en el mismo movimiento, que se le escurra entre las manos. ¿Cuál es según Deleuze el «error» de Fichte?: «Colmar lo que separa». Esto lo hace «dogmático» en sentido deleuziano ${ }^{17}$ : el Yo de Fichte consiste en una identidad sin fisuras consigo mismo. En la identidad absoluta del Yo = Yo todas las determinaciones se

16 Este argumento es válido para Deleuze. Pese a que cierto deleuzianismo considerara la ontología como mera apología del fluir y del devenir puro, Deleuze no desprecia la necesitad de permanencia y perduración de lo que es. Si no, no habría propiamente génesis, sino un fondo indeterminado donde todo se disolvería. Pero el fondo ontológico deleuziano es ideal: también determinable por la determinación.

17 El sentido en que Deleuze usa el término "dogmatismo» no es el mismo que en Fichte. En Fichte, el dogmatismo consiste en afirmar «una cosa en sí abstraída de lo que ocurre en la experiencia» (Fichte, 1970, 188), mientras que para Deleuze se trata de establecer 
disuelven y la determinación como proceso se estanca. Y así liquidaría sus propias producciones. Para que haya génesis, no puede haber puro movimiento, pero tampoco puro ser, tranquila y quieta permanencia y perduración. Si el fundamento es la identidad absoluta, no hay génesis. Y eso es, según Deleuze, lo que ocurre en el poskantismo. Mientras las Ideas deleuzianas hormiguean en la grieta, emergen constantemente sobre los bordes de esta grieta, «saliendo y entrando sin cesar, componiéndose de mil maneras diversas» (Deleuze, 1968, 220), las producciones de Fichte encuentran en el Yo su tranquilo reposo. La ruhende Kraft se develaría como la esencia de la Handlung: «La fuerza no era puesta en la actividad, sino fuerza calma» (Fichte, GA 1/3, 177). En la calma no hay génesis ni producción. Por eso, dice Deleuze, «no es cuestión de colmar lo que no pude ser colmado» (Deleuze, 1968, 220).

Si en la confusión los valores lógicos pierden su carácter genético, ¿cómo puede entonces Fichte haber alcanzado en alguna medida el punto de vista de la génesis? ¿Cómo puede el Yo de Fichte ser al mismo tiempo unidad objetiva interna de determinación, indeterminado y determinable $e$ identidad y confusión de los valores? ¿Cuál es el rol (el valor) de lo indeterminado y de lo determinable si lo único que persiste es una determinación fija? ¿Cómo compatibilizar la crítica y el elogio deleuziano?

La respuesta a esta paradoja está en otra crítica, que Deleuze realiza más adelante (treinta páginas después, pero siempre dentro del capítulo IV «Síntesis ideal de la diferencia»). Allí el cuestionamiento es que el "punto de llegada» del sistema de Fichte es el Yo como «imperativo de orden eminentemente moral» (Deleuze, $1968,254)$. Teniendo en cuenta esta crítica al moralismo fichteano podemos completar el arco de la interpretación deleuziana de Fichte.

En efecto, si bien en un inicio el Yo aparece como unidad interna de la determinación, lo determinable y lo indeterminado, y existe una multiplicidad de determinaciones fruto del proceso de producción que hemos señalado más arriba, el punto de llegada, el destino, es una determinación completa donde todas las determinaciones parciales se reabsorben. El Yo deviene igual al Yo, a través de la superación de su oposición con el no-Yo (en una visión de Fichte teñida de hegelianismo). La determinación se disuelve en el "destino" (la Bestimmtheit en la Bestimmung). La grieta no está colmada (hay límite, hay determinación) pero

«identificación y confusión en la Idea», es decir, identificar y confundir determinación, indeterminable e indeterminado (Deleuze, 1968, 221). 
debe colmarse. El imperativo moral y el dogmatismo se anudan así. Durante la germinación del Yo, el proceso de determinación es evidente: El Yo pone al no-Yo, reflexiona sobre su objeto indeterminado, lo pone como imagen, como cosa real, es así determinado mediatamente por lo que en un inicio era mera indeterminación, etc. Pero en el punto de llegada el no-Yo se reabsorbe necesariamente en el Yo. Así parece afirmarlo Fichte en el Fundamento:

En cuanto el Yo es puesto, toda la realidad es puesta; todo debe ser puesto en el Yo (...); todo debe depender de él. De esta suerte se exige el acuerdo del objeto con el Yo; y es el Yo absoluto el que lo exige precisamente en virtud de su ser absoluto [lo que sigue está en nota]: es el imperativo categórico de Kant (...) ¿Cómo hubiera podido jamás llegar a un imperativo categórico como postulado absoluto de un acuerdo con el Yo puro, sin suponer el ser absoluto del Yo, por el cual todo sería puesto, y por lo menos debería serlo, en la medida en que no lo es? (Fichte, GA I/2, 396 [1975, 123-124]).

El imperativo categórico, la moral, el deber ser, apuntan a una coincidencia absoluta del Yo y el no-Yo. Exigen una reabsorción de la determinación, del límite, del choque, en la pura identidad originaria del Yo. Igualdad, concordancia, acuerdo en el seno del Yo absoluto. Aniquilación del no-Yo y el límite. El primer principio fundamental «absolutamente incondicionado» se revela, hegelianamen$t^{18}{ }^{18}$, como la verdad de los otros, "condicionados», donde éstos encuentran su reposo. La grieta queda colmada, la fuerza se aquieta. Si lo que debe ser (que no es) llega a ser, la fuente de la génesis se seca, ya no hay producción, volvemos al punto de vista del condicionamiento. La tensión entre devenir y ser que mencionamos más arriba, la necesidad de interrupción de la fuerza para que algo sea, y por tanto para que algo se transforme, sólo es pertinente para la naturaleza humana. En el Yo absoluto rige la serena continuidad de la beatitud. El juego de lo indeterminado, la determinación y lo determinable perece en favor de un estado final donde la determinación completa equivale a la más alta indeterminación. Lo determinable, por su parte, ha cumplido ya su función y puede prescindirse de él. Estamos ante «un Yo absoluto, completamente incondicionado y no determinable por algo más elevado» (Fichte, GA I/2, 279). Tal es el destino del Yo, en términos de las Lecciones sobre el destino del sabio: su último fin final es «someter a sí mismo todo lo irracional, dominarlo libremente, y según su propia ley» (Fichte, GA I/3,

18 En un hegelianismo que, creo yo, traiciona el espíritu de Hegel, concibiendo el espíritu absoluto como la disolución de todos los momentos precedentes (en la línea de Kojève y el fin de la historia), cuando en realidad es su eterna vivificación. 
$32[2002,59])$, y de lo que se trata es de determinar los medios para alcanzarlo (Fichte, GA I/3, 27 [2002, 39]). El concepto de tendencia [Streben] no haría otra cosa que apuntar a ese camino, marcar el trayecto necesario hacia el punto de llegada moral, el imperativo categórico del acuerdo absoluto en el Yo.

\section{EI moralismo como actividad}

Así como me parece bella y aguda la interpretación de Deleuze de Fichte en términos de los tres valores lógicos y el poder genético que de su dinámica interna se deriva, considero que su crítica al carácter moral de la filosofía de Fichte es un desacierto. Lejos de taponar el movimiento genético característico de la triplicidad de los valores lógicos, el moralismo de Fichte los efectiviza. En efecto, la interpretación que acabamos de esbozar en torno a una absorción de todo límite en la unidad indeterminada del Yo absoluto sólo se sostiene en una lectura sesgada de los textos de Fichte. La frase completa de las Lecciones citada más arriba es: «El último fin final del hombre es someter a sí mismo todo lo irracional, dominarlo libremente y según su propia ley. Este fin final es completamente inalcanzable y tiene que permanecer eternamente inalcanzable» (Fichte, GA I/2, 32 [2002, 59], el subrayado es mío $)^{19}$. En la página siguiente del Fundamento a aquella en la que define su imperativo categórico, Fichte aclara que su concepto de tendencia, lejos de apuntar a la identidad efectiva de las determinaciones del Yo absoluto, la conjura: "La actividad del Yo relacionada con aquel no es una determinación (a la identidad efectiva), sino solamente un apetito, una tendencia hacia la determinación» (Fichte, GA I/2, 397 [1975, 124]). No es una determinación a la identidad efectiva, sino una tendencia hacia la determinación. La tendencia como realización efectiva de la identidad sería una "tendencia indeterminada» y por lo tanto no podría llamarse con propiedad tendencia. Y es indeterminada «en general» en tanto escapa a la determinabilidad: «La tendencia indeterminada en general escapa a toda determinabilidad (y por lo tanto no debería llamarse alegremente tendencia, ya que no tiene objeto)» (Fichte, GA I/2, 403 [1975, 129]). Tanto la determinación pura (la identidad efectiva) como la indeterminación resultan indeseables para Fichte. Es indispensable su imbricación, y para ello es necesario recurrir a la determinabilidad. Pero tanto la escisión como la exterioridad de los términos son características de la parte teórica de la Doctrina de la ciencia. Para que la unidad interna de los valores lógicos sea posible, debe

19 Para una lectura distinta del rol del fin final en Fichte, $c f r$. Oncina Coves, 1996, 83: "la revolución es el medio cultural para alcanzar el fin final". 
intervenir el moralismo de Fichte. Sin imperativo categórico nos limitaríamos a la parte teórica del Fundamento:

No podíamos concebir de ninguna otra manera la representación en general como posible, sino presuponiendo que se daba un choque en la actividad del Yo proyectada el infinito y a lo indeterminado. Así, pues, el Yo como Inteligencia en general es dependiente de un No-Yo indeterminado y hasta ahora completamente indeterminable (Fichte, GA I/2, 386-387 [1975, 116]).

No podiamos, hasta ahora. Fichte, al inicio de la parte práctica del Fundamento de toda la Doctrina de la Ciencia de 1794 señala las limitaciones de la perspectiva puramente teórica. El punto de vista teórico, el punto de vista del yo limitado por el no-Yo es el punto de vista de la representación, no menos criticado por Fichte que por Deleuze. Pero este limitar fijo, lejos de ser un acto de libertad del Yo, es por el contrario el signo de su dependencia respecto de un no-Yo indeterminado y (hasta ahora) indeterminable. Implica una «separación» entre la determinación (Yo como inteligencia, Yo empírico) y lo indeterminado (no-Yo) que, desde este punto de vista es completamente indeterminable.

Deleuze prejuzga el sentido de la moral en Fichte desde una perspectiva nietzscheana. Pero las páginas de su Nietzsche y la filosofía de 1962 muestran que el rol de la moral en la interpretación deleuziana de Nietzsche nada tiene que ver con Fichte. En Nietzsche, la moral implica una oposición fija entre el mundo de la existencia y el "mundo verdadero", y una concepción sustancialista de los términos en juego ${ }^{20}$. Pero justamente la Thathandlung de Fichte pone en jaque esa oposición fija y esa concepción sustancialista: "[la Thathandlung] se halla justamente en el punto que enlaza ambos mundos» (Fichte, GA I/4, 221). La moral fichteana, por tanto, implica la superación de la moral que Deleuze critica, convirtiéndose en todo caso en «la verdadera moral que se burla de la moral» (Deleuze, 1962, 103). Y en, efecto, la parte práctica del Fundamento deshace las oposiciones aparentemente fijas que habían sido establecidas en la parte teórica. $\mathrm{El}$ «hasta ahora» de Fichte marca, precisamente, el pasaje a la parte práctica. $\mathrm{O}$ el pasaje a la «verdadera moral».

20 «La oposición del conocimiento y la vida, la distinción de los mundos, revelan su verdadero carácter: es una distinción de origen moral» (Deleuze, 1962, 109). «La distinción concreta entre las fuerzas, la diferencia original entre las fuerzas cualificadas (lo bueno y lo malo), es reemplazada por la oposición moral entre fuerzas sustancializadas (el bien y el mal)» (Deleuze, 1962, 142). 
A partir de ahora, el No-Yo indeterminado (multiplicidad kantiana, cosa en sí kantiana) devendrá determinable. Pero no devendrá completamente determinado, ya que si fuera completamente determinado sería lo infinito puro. No sólo no existiría conciencia, sino que tampoco existiría ser, no existiría realidad. El ponerse de los limites es una actividad necesaria del absoluto. La actividad del Yo es un limitarse, un autolimitarse (limitarse, superar el límite). Sin límite no habría conciencia, no habría ser. No se trata de alcanzar efectivamente la identidad pura en el Yo, sino de una tendencia infinita. En ese sentido es lo determinable el concepto clave.

Los opuestos deben ser conjuntados [zusammengefaßst] en el concepto de la mera determinabilidad (y no en el de la determinación) (...). Si entre los opuestos (...) se pone el límite como sólido, fijo e inmutable, entonces los dos términos son conciliados por determinación, pero no por determinabilidad (...). La determinabilidad es la Idea -inalcanzable por este camino- de la determinación, pero no la misma determinación (...). La imaginación es una facultad que oscila pendularmente en el punto medio que hay entre la determinación y la no determinación, entre lo finito y lo infinito (Fichte, GA I/2, 359-360 [1975, 92-93]).

Es lo determinable lo que expresa la dinámica, la actividad que está en el fundamento de las supuestas determinaciones fijas. Es el movimiento de la determinabilidad lo que hace del Yo un actuar no una sustancia. Por eso Fichte caracteriza al objeto del idealismo como lo determinable (Fichte, GA 1/4, 189). "iAlegrémosnos de sentir esa fuerza, y de que la tendencia sea infinita!» (Fichte, GA I/3, 68). Sin determinabilidad no sólo el no-Yo es indeterminado: también el Yo (yo puro, yo absoluto, sin límite, sin determinación). Por eso la determinabilidad es un concepto central, clave para la comprensión de la Thathandlung como esencia del Yo. El Yo no es otra cosa que su actuar, y el actuar no es otra cosa que la intrinsecación de lo indeterminado, lo determinable y la determinación. Así, la interpretación deleuziana de Fichte va al corazón de la Doctrina de la ciencia.

De la misma manera, la triplicidad de valores lógicos va al corazón de la filosofía de Deleuze. En efecto, los dos conceptos que dan nombre a su obra magna, Diferencia y repetición, tienden a fusionarse. Deleuze dice que el fin que persigue su filosofía es «instaurar una adecuación cada vez más perfecta entre la 
diferencia y la repetición» (Deleuze, 1968, 39) ${ }^{21}$. La diferencia sería abstracta, indeterminada (indiferencia) si no fuera al mismo tiempo repetición, mediante la cual entra en un proceso de determinación. Pero la diferencia no es sustrato y la repetición una acción extrínseca, mecánica. La diferencia se diferencia como repetición. Y esto porque la diferencia se repite internamente en sus tres valores: la determinación, lo determinable y lo indeterminado. La repetición no es perpetuación de lo mismo, de lo idéntico, no es monótono reiterar lo dado, sino proceso de producción. Y esto porque lo que repite es la dinámica de los valores internamente diferenciados.

Sin embargo, cuando Deleuze dice que el fin que persigue su filosofía es instaurar una adecuación cada vez más perfecta entre la diferencia y la repetición, podría ser objeto de la misma crítica que Deleuze le hace a Fichte: una tendencia a la igualación de las diferencias en un estado final de beatitud. ¿Moralismo de Deleuze? En efecto, pero verdadero moralismo: ni en Fichte ni en Deleuze, se trata de estados, sino de actividad. Por eso decía Deleuze en su último texto que en Fichte «la actividad misma no remite ya a un ser, sino que no cesa de ponerse en una vida» (Deleuze, 2003, 361) y eso era para él la beatitud de la inmanencia. No implica quietud, porque no es ser sino perpetuo ponerse. La diferencia y el Yo no deja de ponerse. La perfección de la adecuación no es el fin de la actividad: es la actividad misma. Así la repetición de la diferencia teje el proceso de diferenciación que constituye lo real. De la misma manera en que la determinabilidad infinita del Yo absoluto fichteano pone sin cesar una determinación (límite) como actividad de ser para sí lo que es en $s^{i 22}$.

\section{Las paradojas del límite}

La estrecha afinidad que así establecemos entre Fichte y Deleuze no carece de peligros para la interpretación y especificidad de sus pensamientos. En otra parte (Ferreyra, 2014a) señalábamos que el intento de J.-Ch. Goddard de establecer una excesiva coincidencia entre ambos autores afectaba los fundamentos de la filosofía de Fichte (Goddard, 2008). Pero esta objeción no responde en sí a una

21 «La repetición comprende la diferencia (no como una variante accidental o extrínseca, sino como su corazón)» (Deleuze, 1968, 370) mientras que «la repetición «hace» la diferencia» (374).

22 «Lo que existe para un ser racional, existe en él. Pero en él no hay nada salvo lo que procede de un actuar sobre sí mismo», Fichte, GA I/3, 313. 
nociva influencia de la perspectiva deleuziana en la doctrina de Fichte, sino a problemas inherentes a la interpretación que Goddard sostiene de Deleuze, según la cual su concepción de lo trascendental no logra más que «esbozar un espacio extremadamente abierto y sin relieve ni modulación» (Goddard, 1999, 133). El fondo ontológico deleuziano sería así trágica y dolorosamente fallido (Ibid). Esa lectura (por cierto habitual en los estudios deleuzianos) implica una dominancia de lo indeterminado sobre los otros valores lógicos. Esa avería ontológica es la que subrepticiamente, en su artículo de 2008, Goddard traslada a Fichte en busca de la actividad pura del Yo como beatitud de la inmanencia (contra las interpretaciones quietistas de la ontología fichteana). La afinidad que presentamos aquí va en otro sentido: ni el fondo ontológico deleuziano ni la actividad pura del yo son meramente abiertas, infinitas, absolutas, ilimitadas. Lo indeterminado no devora la determinación. Y no lo hace, por la importancia que tiene en ambos autores el valor de lo determinable. La interpretación que sostenemos en estas páginas no es por tanto susceptible de las objeciones que hemos realizado al afinismo de Goddard.

El problema es que para apuntalar esta interpretación, reivindicamos el concepto fichteano de límite. Sostuvimos que la coincidencia absoluta del Yo y el no-Yo es inalcanzable e indeseable. Pero para ello es necesario sostener el tercer principio del Fundamento de toda la Doctrina de la Ciencia: «el yo y el no-Yo son puestos como divisibles» (Fichte, GA I/2, 270). Divisibilidad (Thailbarkeit) y determinabilidad están ligados. Lo cual plantea un obstáculo teórico desde el punto de vista deleuziano, ya que el límite es una de las formas de la negatividad (a la cual Deleuze identifica como el gran enemigo de la filosofía de la diferencia, aunque, como veremos, la cuestión es conceptualmente más compleja que un liso rechazo).

Se suele asignar un cambio filosófico considerable al pasaje entre los momentos representados por el prekantismo y el poskantismo. El primero se definiría por el negativo de limitación, el otro por el negativo de oposición. Uno por la identidad analítica, el otro por la identidad sintética. Uno desde el punto de vista de la sustancia infinita, el otro desde el punto de vista del Yo finito (...) Para una filosofía de la diferencia, poco importa que lo negativo sea concebido como negativo de limitación o de oposición, y la identidad como analítica o sintética, a partir del momento en que la diferencia está reducida a lo negativo o subordinada a lo idéntico (Deleuze, 1968, 81). 
Poner el acento en el concepto fichteano de límite no sólo pondría a Fichte en la lista de los condenados por Deleuze en tanto "filósofo de la negatividad», sino que también implicaría un retroceso en el buen sentido de la historia de la filosofía, en tanto el límite es característico del prekantismo. Para pertenecer de pleno derecho al poskantismo, el límite de Fichte debería estar subordinado a la oposición (como en Hegel el pasaje inmediato que implica el límite en la doctrina del ser a la mediación por las determinaciones de la reflexión en la doctrina de la esencia). Toda la fundamentación encontraría su eje en el segundo principio, al cual quedarían subordinados tanto el tercero (el límite) como el primero (el Yo absoluto como punto de vista de la sustancia infinita). Sin embargo, el segundo desplazamiento que señala Deleuze va en sentido inverso: el pasaje del punto de vista de la sustancia infinita al del Yo finito sólo es posible mediante la limitación del $Y o^{23}$.

Esta incongruencia entre los desplazamientos enumerados, lejos de afectar a Deleuze, apuntala su argumento: el pasaje importa poco. El pasaje de la limitación a la oposición no implica un cambio filosófico considerable. Tampoco el del punto de vista de Dios al punto de vista del Yo. Por el contrario, se trata de términos en relación recíproca en tanto derivan de un mismo fundamento: «la unicidad y la identidad de la sustancia divina son en realidad el único garante del Yo uno e idéntico, y Dios se conserva mientras nos quedamos con el Yo» (Deleuze, 1968, 81). Tanto limitación como oposición aparecen como principios de la filosofía de Fichte, pero en la medida en que son expresiones de la Thathandlung que es su fundamento. De la misma manera, tampoco es el límite en sí mismo lo que Deleuze rechaza. Sin una forma del límite (lo determinable, la fisura, la grieta donde hormiguean las Ideas) la ontología deleuziana remitiría a un fondo indiferenciado y la filosofía de la diferencia no sería otra cosa que una filosofía de la indiferencia. Tampoco la oposición es rechazada en sí misma por Deleuze (la acepta explícitamente en tanto derivada ${ }^{24}$ ). Más aún, ni siquiera la negatividad, que muchos comentaristas suponen el enemigo último de la filosofía de Deleuze,

23 El tercer rasgo (pasaje de lo analítico a lo sintético) no es menos problemático en Fichte ya que él dice explícitamente que el poner absoluto del Yo «es tético y no sintético» (Fichte, 1970, 255). El propio Deleuze reconoce que uno de los rasgos del poskantismo es el esfuerzo por alcanzar la "transformación del juicio hipotético en juicio tético» (Deleuze, 1968, 254). Pero justamente, el juicio tético no es ni el analítico ni el sintético, sino el fundamento de éstos. Para un análisis del rol del juicio tético en Fichte, en referencia al desplazamiento señalado por Deleuze, $c f r$. Ferreyra, 2014b.

24 «El descubrimiento de una pluralidad de oposición coexistentes, en todo dominio, no es separable del descubrimiento más profundo, el de la diferencia» (Deleuze, 1968, 263). 
es impugnada en sí misma y de hecho cierta forma de la negatividad no sólo puede sino que debe existir para la afirmación de la diferencia: «el ser es también no-ser (...) Un (no)-ser que es el elemento diferencial donde la afirmación, como afirmación múltiple, encuentra el principio de su génesis» (Deleuze, 1968, 89). Lo que está puesto en cuestión no es la negatividad en sí misma, sino su función.

Ni el límite, ni la oposición, ni la negatividad son lo filosóficamente relevante. Tampoco Dios ni el Yo. Se les suele otorgar una importancia filosófica considerable, pero en realidad importan poco. No es allí donde se define la cuestión. La experiencia crucial radica en establecer cuál es el fundamento del cual dependen: ¿se afirma la diferencia en sí misma o se la subordinada a lo idéntico? He allí la cuestión. La identidad como fundamento es el único adversario de la filosofía de Deleuze, porque en ella todas las diferencias perecen. La cuestión se reduce entonces a una única pregunta: ¿está la diferencia subordinada a lo idéntico? En Fichte esto sólo es así si se concibe que el Yo = Yo del primer principio es el fundamento de toda su filosofía. En ese caso, la identidad fundamentaría toda la Doctrina de la ciencia. Pero, como hemos mostrado, el Yo fichteano no es un sujeto uno e idéntico. El Yo de Fichte no es sustancia, sino actividad ${ }^{25}$. Poco importa en Fichte la limitación o la oposición, Dios o el $\mathrm{Yo}^{26}$, porque todas las figuras de la Doctrina de la ciencia están subordinadas a la Thathandlung.

\section{Conclusión}

En conclusión, ¡es la Thathandlung un modo de subordinar la diferencia a la identidad? El concepto de lo determinable nos permite responder a esta pregunta en forma negativa. Sin lo determinable las determinaciones serían fijas, sustanciales, y el Yo no sería actividad, Thathandlung, sino sustrato, Yo = Yo como identidad última. Pero lo determinable, como hemos mostrado, es un concepto

25 «Se ha argumentado contra la Doctrina de la Ciencia el que pusiera como fundamento de la filosofía un Yo en tanto sustrato existente sin la intervención del Yo (un Yo como cosa en sí) (...) Esta gente no puede comenzar nada sin sustrato, porque les es imposible elevarse desde el punto de vista de la experiencia común al punto de vista de la filosofía» (Fichte, GA $\mathrm{I} / 3,313)$.

26 Varios comentaristas sostienen la continuidad del pensamiento de Fichte más allá del pasaje del Yo a Dios: «Una de las apuestas de la lectura que sugerimos aquí es establecer la unidad originaria de la filosofía del yo y la filosofía del Absoluto, mediante la continuidad del proyecto fichteano de un lado al otro de la línea de fractura constituida por la polémica del ateísmo,", Goddard, 1999, 7. Cfr. Lauth, 1968, 28. 
clave de la Doctrina de la ciencia. No por nada en la Primera introducción a la Doctrina de la ciencia establecía a lo determinable como el objeto propio del idealismo (Fichte, GA I/4, 190). Como en Deleuze, lo determinable es lo que hace funcionar la máquina entera: «Es la diferencia, o la forma de lo determinable, lo que hace funcionar al pensamiento, a la máquina entera de lo indeterminado y de la determinación» (Deleuze, 1968, 354, yo subrayo). Lo determinable es lo que permite que la grieta en la Idea no trasforme a sus tres valores en términos extrínsecos, sino que en ella "las Ideas hormigueen, surgiendo constantemente sobre sus bordes» (Deleuze, 1968, 220). En las ontologías de Fichte y Deleuze, el ser determinado no es una finitud que niega lo infinito, sino eterna afirmación de lo infinito como actividad de diferenciarse, determinándose a través de su propia limitación. La Thathandlung, pensada como afirmación de la diferencia, principio de génesis de lo real y de su dinamismo inagotable, resuelve «el desconcierto más sorprendente y ancestral»: cómo es posible que el yo sea absolutamente libre y limitado al mismo tiempo, finito e infinito, esto es necesariamente finito e infinito. 


\section{Bibliografía}

(En los casos en los que se utiliza una traducción, se señala la misma entre corchetes; en caso contrario, la traducción es mía)

Acosta, E. (2009) «Pensar tres absolutos: Kant y Fichte, Deleuze y Boeder», en López, D. (ed.) Experiencia y Límite : Kant Kolloquium (1804- 2004), Santa Fe: Ediciones de la Universidad Nacional del Litoral.

Breazeale, D. (2013), Thinking Through the Wissenschaftslehre: Themes from Fichte's Early Philosophy, Oxford: Oxford University Press.

Deleuze, G. (1962) Nietzsche et la philosophie, Paris: PUF.

- (1968) Différence et répétition, Paris: PUF.

— (2003) Deux régimes de fous, Paris: Minuit.

Ferreyra, J. (2012) «Ideas, from Hegel to Deleuze», Comparative and Continental Philosophy, 4.1, pp. 93-104.

- (2014a) «Fichte y Deleuze: potencias y paradojas del afinismo radical», Revista de Estud(i)os sobre Fichte [En línea], 8 | 2014, Publicado el 21 septiembre 2014, consultado el 22 septiembre 2014. EuroPhilosophie Editions. URL : http://ref.revues.org/525.

— (2014b) «El juicio tético en Fichte: roce y traición en el verdadero movimiento del pensamiento», en Ferreyra, J. y Soich, M. (eds), Deleuze y las fuentes de su filosofia, Buenos Aires: La Almohada, pp. 105-114.

- (2014c) «El nacimiento en la biopolítica: la génesis de la vida en Hegel y Deleuze», Daimon, Revista Internacional de Filosofía, Universidad de Murcia, no 61, pp. 149-164.

Fichte, J.G. (1964), Gesamtausgabe der Bayerischen Akademie der Wissenschaften, Reihe I: Werke, Band 2, Stuttgart: Frommann.

- (1966), Gesamtausgabe der Bayerischen Akademie der Wissenschaften, Reihe I: Werke, Band 3, Stuttgart: Frommann.

- (1970), Gesamtausgabe der Bayerischen Akademie der Wissenschaften, Reihe I: Werke, Band 4, Stuttgart: Frommann.

- (1975), Doctrina de la ciencia, trad. Cruz Cruz, J. Buenos Aires: Aguilar.

- (1994), Fundamento del derecho natural según los principios de la doctrina de la ciencia, trad. Villacañas Berlanga, J. Ramos Valera, M. y Oncina Coves, F., Madrid: Centro de Estudios Constitucionales.

- (2002), Algunas lecciones sobre el destino del sabio, (trad. Oncina Coves, F. y Ramos Valera, M., Madrid: Itsmo. 
GodDARD. J.-Ch. (1999), La philosophie fichtéenne de la vie, Paris: Vrin.

— (2008), «Commentaire du $\S 16$ de la Critique de la raison pure», in Vaysse, Jean-Marie (ed.), Kant, Paris: Cerf, pp. 129-148.

LaUth, R. (1968), La filosofia de Fichte y su significación para nuestro tiempo, trad. Navarro, B., UNAM: México.

Oncina Coves, F. (1996), "El iusnaturalismo agónico: La teoría fichteana del contrato", en López Domínguez, V. (ed.), Fichte, 200 años después, Madrid: Editorial Complutense.

Rametta, G. (2010), «Consciousness. A Comparison Between Fichte and the Young Sartre in a Bio-Political Perspective», en Waibel, V. Fichte and the Phenomenological Tradition, de Gruyter: New York.

Zöller, G. (1998), Fichte's Transcendental Philosophy, Cambridge: Cambridge University Press.

Enviado: 20/02/2015

Aceptado: 13/05/2015

Este trabajo se encuentra bajo una licencia de Creative Commons Reconocimiento-

NoComercial-SinObraDerivada 4.0

\section{(1) 9 \\ BY NO ND}

ÉNDOXA: Series Filosóficas, n. ${ }^{\circ}$ 36, 2015, pp. 161-180. UNED, Madrid 
